\title{
Stability of Copper Atoms Embedded in Sodium-Chloride Crystals
}

\author{
Miou Furuya ${ }^{1, *}$, Soh Ishii ${ }^{2}$, Yoshio Takahashi ${ }^{3}$, Shin-ichiro Nagasaka ${ }^{3}$, \\ Takehisa Yoshinari ${ }^{3}$, Yoshiyuki Kawazoe ${ }^{2}$ and Kaoru Ohno ${ }^{1}$ \\ ${ }^{1}$ Department of Physics, Graduate school of Engineering, Yokohama National University, Yokohama 240-8501, Japan \\ ${ }^{2}$ Institute for Materials Research, Tohoku University, Sendai 980-8577, Japan \\ ${ }^{3}$ Department of Physics, Faculty of Science, Yamagata University, Yamagata 990-8560, Japan
}

\begin{abstract}
Because it has been pointed out by Nagasaka et al. that the optical absorption spectrum of copper atoms embedded in alkali-chloride crystals may depend on the position of the copper atoms, we calculate the relation between the optimal position and the total energy of copper atom embedded in $\mathrm{NaCl}$ crystal by means of the first-principles pseudopotential plane-wave-expansion method. These results shows that most stable position of embedded $\mathrm{Cu}^{+}$ion in these alkali halide system are not the substitutional on-center site but off-center site along $\langle 111\rangle$ axis.
\end{abstract}

(Received December 17, 2003; Accepted January 16, 2004)

Keywords: ligand field, first-principles method, optimal position

In order to study optical properties of transition metal ions, alkali halide crystals doped with transition metal atoms and compounds play an important role. Because alkali halide crystals have a wide energy gap, these crystals are transparent around optically visible ranges. So one may directly investigate optical properties of impurity ions embedded in these crystals.

Nagasaka et al. has proposed that a model for the impurity $\mathrm{Cu}^{+}$ions in alkali halide crystals in which a charge-transfer excitation between the impurity ion and the neighboring halogen ions is assumed to play an essential role in the offcenter instability. ${ }^{1,2)}$ They have also measured optical absorption spectrum of alkali ( $\mathrm{Na}$ and $\mathrm{K}$ ) halide crystals doped with some impurity copper atoms. ${ }^{3)}$ This experiment showed that the absorption peak intensity at the lowest photon energy $(\sim 4.8 \mathrm{eV})$ decreases when the $\mathrm{NaCl}$ sample is cooled down, and hardly changes when the $\mathrm{KCl}$ sample is cooled down. This fact suggests that in the $\mathrm{NaCl}$ crystal, the optical absorption peak is assigned to $3 d^{10} \rightarrow 3 d^{9} 4 s$ transition of the $\mathrm{Cu}^{+}$ion near the normal lattice site (referred to as the on-center site) and that this transition is a parityforbidden at the on-center site but is partially allowed by the lattice vibration. So they concluded that $\mathrm{Cu}^{+}$ion is stable at the on-center site in $\mathrm{NaCl}$. In contrast, in the $\mathrm{KCl}$ crystal $\mathrm{Cu}^{+}$ ion is located at a sligntly displaced position (referred as offcenter site), away from the on-center sites.

In this paper, we investigate the relation between the optimal position and the total energy of the embedded copper atom in $\mathrm{NaCl}$ crystal to study whether these systems have really the different optimal positions or not.

We carry out the structural optimization for these systems in the following way. We assume the $2 \times 2 \times 2$ facecentered-cubic unit cell of the $\mathrm{NaCl}$ crystal structure, and replace one $\mathrm{Na}^{+}$ion with one copper ion. The copper ion is placed either at the on-center or off-center sites along the $\langle 100\rangle,\langle 110\rangle$, and $\langle 111\rangle$ axis. We optimized all atomic positions inside the unit cell by means of the standard $a b$ initio ultrasoft-pseudopotential approach (VASP) ${ }^{4,5)}$ with the 17 Ry cutoff energy for the plane waves (PW's) simulated

*Graduate Student, Yokohama National University annealing the conjugate-gradient algorithm. From this calculation, we have found the following facts:

i When the embedded copper atom is put at the on-center site, it does not move. That is, the on-center site is at least locally stable.

ii If the copper atom is put on some particular axis like $\langle 100\rangle,\langle 110\rangle$ and $\langle 111\rangle$, then the copper atom roams and moves toward the most stable position along the same axis.

iii The copper atom tends to move toward to positions on $\langle 111\rangle$ axis when it is put at an off-center site that is not on a particular axis. And then it moves as the same as (ii).

iv The axis having the most stable position is $\langle 111\rangle$ and the next stable axis is $\langle 110\rangle,\langle 100\rangle$, and so on. The on oncenter site is not the most stable position.

Because the energy optimize calculation is performed with fixed crystal symmetry, the copper atom at on-center site cannnt move off from the particularly axis. Moreover, these calculations do not take into account the thermodynamic effect, so that this optimization is limited to the absolute zero temperature only. But in the $\mathrm{NaCl}: \mathrm{Cu}$ systems, difference in the total energy between the on-center site and the most

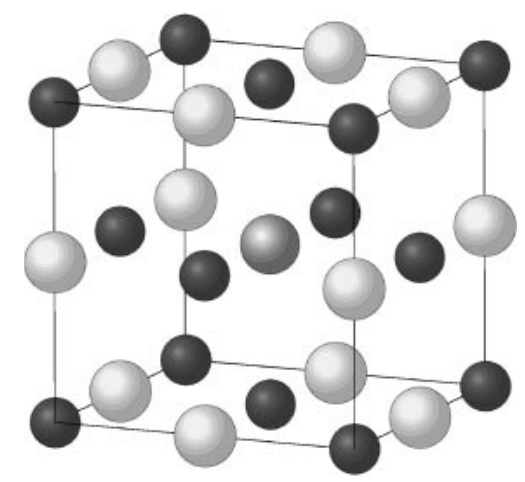

Fig. 1 Schematic illustration of the unit cell. It is a $2 \times 2 \times 2$ facecentered-cubic lattice of the sodium-chloride crystal structure with the center atom replaced by copper atom. The white spheres represent sodium atoms, and black and silver spheres represent chlorine and copper atoms, respectively. 
$\mathrm{NaCl}: \mathrm{Cu}<100>$

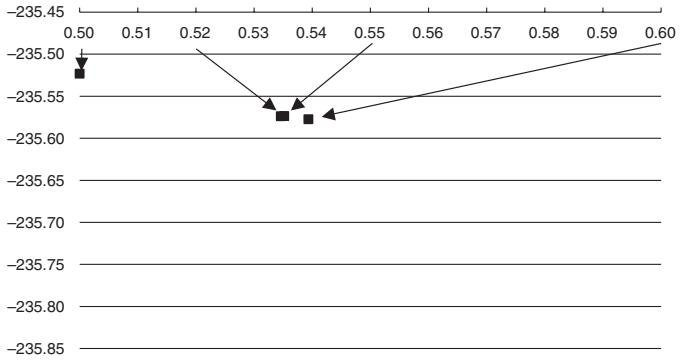

$-235.85$

60

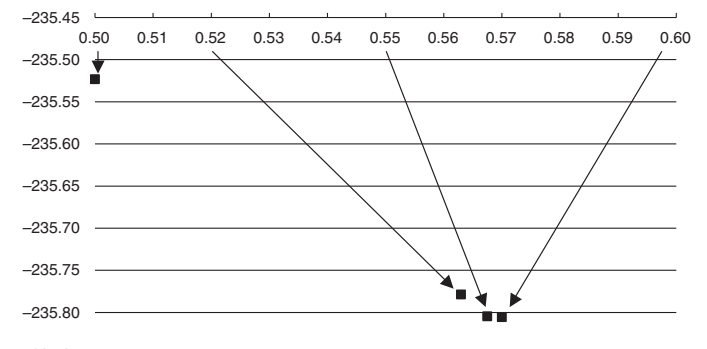

$-235.85$

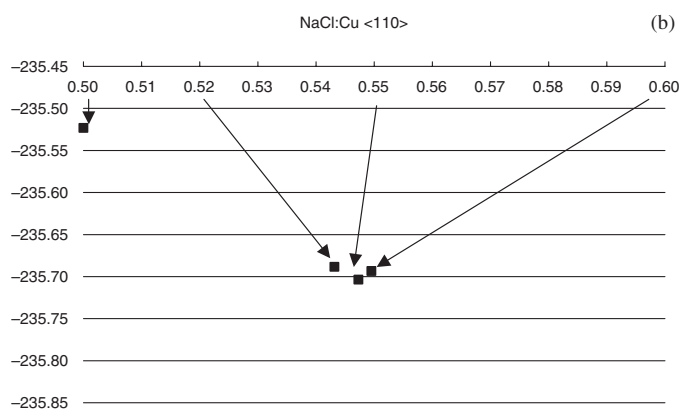

(b)

Fig. 2 These figures represent optimal positions and total energies in eV after the ionic relaxation in the $\mathrm{NaCl}: \mathrm{Cu}$ system. The arrow shows the direction of the movement of copper atom that was put on various positions. The vertical axis represents the total energy and the horizontal axis represents the distance away from the on-center site. The on-center site is $(0.5,0.5,0.5)$. The displacements of copper atom along (a) the $\langle 100\rangle$ axis, (b) $\langle 110\rangle$ axis and (c) $\langle 111\rangle$ axis are represented in separate figures.

stable site along $\langle 111\rangle$ is no less than $0.3 \mathrm{eV}$. So we need not take into account the thermal vibration around the room temperature.

It is worth mentioned that ionic sizes are almost same for $\mathrm{Na}^{+}$and $\mathrm{Cu}^{+}$ions. But the ionic size of $\mathrm{Cu}^{+}$ion is smaller than that of $\mathrm{K}^{+}$ion (the Pauling ionic radii of $\mathrm{Li}^{+}, \mathrm{Na}^{+}, \mathrm{K}^{+}$ and $\mathrm{Cu}(\mathrm{I})^{+}$are $0.60,0.95,1.33$ and 0.96 , respectively). Therefore we can imagine that copper ion embedded in $\mathrm{NaCl}$ crystal is more tight compared with that in $\mathrm{KCl}$ crystal and is more flexible compared with that in $\mathrm{LiCl}$ crystal. Therefore one may say that $\mathrm{NaCl}: \mathrm{Cu}$ system is in the middle of these $\mathrm{LiCl}: \mathrm{Cu}$ and $\mathrm{KCl}: \mathrm{Cu}$ system. It is left for the future study to compute their optical absorption spectra and compare with experiments.

\section{Acknowledgements}

The authors acknowledge the support of the HITACHI
SR8000 supercomputing facilities that are owned by the Computer Science Group at the Institute for Materials Research, Tohoku University.

\section{REFERENCES}

1) S. Nagasaka: J. Phys. Soc. Japan 50 (1981) 1570-1578.

2) S. Nagasaka: J. Phys. Soc. Japan 51 (1982) 895-905.

3) A handout IMR (Institute for Materials Research, Tohoku Univ.) -SEMINAR on July 21, 1998.

4) G. Kresse and J. Furthmuller: Compt. Mater. Sci. 15 (1996) 6.

5) G. Kresse and J. Furthmuller: Phys. Rev. B 54 (1996) 169.

Note added in proof: We have performed similar calculation also for $\mathrm{LiCl}$ : $\mathrm{Cu}$ and found that the result is qualitatively similar to the present case of $\mathrm{NaCl}: \mathrm{Cu}$. The optical position of $\mathrm{Cu}$ ion along the $\langle 111\rangle$ axis in the $\mathrm{LiCl}: \mathrm{Cu}$ case is, however, $(0.56,0.56,0.56)$ instead of the present $(0.57,0.57,0.57)$. 